\title{
Plane turbulent buoyant jets. Part 1. Integral properties
}

\author{
By NIKOLAS E. KOTSOVINOS † AND E. J. LIST \\ W. M. Keck Laboratory of Hydraulics and Water Resources, \\ California Institute of Technology, Pasadena
}

(Received 28 January 1976 and in revised form 9 June 1976)

An integral technique suggested for the analysis of turbulent jets by Corrsin \& Uberoi (1950) and Morton, Taylor \& Turner (1956) is re-examined in an attempt to improve the description of the entrainment. It is determined that the hypothesis of Priestley \& Ball (1955), that the entrainment coefficient is a linear function of the jet Richardson number, is reasonable, and that two empirically determined plume parameters are sufficient to describe the transition of buoyant jets to plumes. The results of a series of experiments in which both time-averaged velocity and time-averaged temperature profiles were recorded in a substantial number of plane turbulent buoyant jets of varying initial Richardson numbers are used to verify the basic ideas. In addition, measurements of the mean tracer flux in a series of buoyant jets indicate that as much as $40 \%$ of the transport in plumes is by the turbulent flux.

\section{Introduction}

Turbulent buoyant jets are of interest to people concerned with the environmental problems resulting from the discharge of pollutants into the atmosphere and oceans. The most common example, of course, is the ubiquitous smoke stack, but more recently the use of multiport diffusers to release power-plant discharges and treated municipal and industrial waste into coastal waters has resulted in a renewed interest in two-dimensional buoyant jets. The buoyant jet formed when the individual jets of a multiport diffuser merge is a good approximation to a plane jet, and hence an understanding of the entrainment and mixing processes in such a jet is necessary to permit optimal design of such diffusers.

A substantial number of experimental investigations of turbulent plane jets without buoyancy have been performed (for a review see Kotsovinos 1975). In many of these studies both velocity and tracer-concentration fields have been measured and data have been collected concerning the lateral and axial distributions of mean velocity and concentration. However, for plumes, or jets with any degree of buoyancy, there have apparently been only two studies. Rouse, Yih \& Humphreys (1952) investigated both the velocity and the temperature field above a line fire. Their instrumentation was crude and the results they

$\dagger$ Present address: School of Engineering, University of Patras, Greece. 
obtained were at variance with what was expected on the basis of a similar study of a point fire. More specifically, their experimental results showed the apparent half-width of the mean velocity profile of the plume to be larger than the halfwidth of the mean temperature profile. Since it is well known that for plane jets the opposite is true, and that for axially symmetric jets and plumes the opposite is also true, some debate has ensued as to the validity of their results. Lee \& Emmons (1961) measured only temperature (and not velocity) profiles above a line fire so their results cannot be compared with those of Rouse et al. So far as is known, no further experimental studies of two-dimensional plumes, or jets with buoyancy in transition to plumes, have ever been performed. Thus, while there is a substantial amount of data available describing the basic features of jets the only available results describing buoyancy-driven flows are limited and of dubious quality.

The purpose of the study described here was to make a systematic investigation of buoyancy-driven turbulent flows, especially with regard to the entrainment and mixing processes that result in the dilution of the initial jet discharge. An organized series of experiments has been performed using turbulent jets with varying initial levels of buoyancy in order to obtain some understanding of the role of buoyancy in the flow mechanics. In this paper we describe the integral properties of the flow; results on turbulent fluctuations are the subject of part 2.

\section{The mechanics of jets and plumes}

\section{Basic definitions and previous work}

The flows induced by vertical turbulent line jets with varying levels of buoyancy flux were studied. The fluids are assumed to be incompressible and the ambient fluid to be homogeneous in density and to have only motion induced by the jet itself. A flow geometry is assumed in which an $x, y$ co-ordinate system is oriented with the $x$ axis vertically upwards and coincident with the jet axis. Fluid velocities in the $x$ and $y$ directions are denoted by $u(x, y)$ and $v(x, y)$ respectively. Timeaveraged mean velocities are denoted by an overbar and the mean vertical velocity on the jet axis by $\bar{u}_{m}(x)$. The difference between the time-averaged local fluid density at any point $\bar{\rho}(x, y)$ and the ambient density $\rho_{a}$ is small, so that

$$
\left|\bar{\rho}(x, y)-\rho_{a}\right| / \rho_{a} \ll 1
$$

in the buoyant jet. Thus there is little error in using the ambient density $\rho_{a}$ to replace the local density $\bar{\rho}(x, y)$ in the description of the inertia force. However, the difference $\bar{\rho}(x, y)-\rho_{a}$ is important in the description of the gravitational body forces.

It is further assumed that the flow is fully turbulent and that the viscous and molecular transport of momentum and tracer respectively can be neglected relative to the turbulent transport. With these definitions and assumptions the time-averaged equations for two-dimensional incompressible motion of a turbulent fluid with small density variations are the conservation of mean volume flux

$$
\partial \bar{u} / \partial x+\partial \bar{v} / \partial y=0
$$


the conservation of mean momentum

$$
\begin{gathered}
\bar{u} \frac{\partial \bar{u}}{\partial x}+\bar{v} \frac{\partial \bar{u}}{\partial y}=-\frac{1}{\rho_{a}} \frac{\partial \bar{p}}{\partial x}-\frac{\bar{\rho}-\rho_{a}}{\rho_{a}} g-\frac{\overline{\partial u^{\prime 2}}}{\partial x}-\frac{\overline{\partial u^{\prime} v^{\prime}}}{\partial y} \\
\bar{u} \frac{\partial \bar{v}}{\partial x}+\bar{v} \frac{\partial \bar{v}}{\partial y}=-\frac{1}{\rho_{a}} \frac{\partial \bar{p}}{\partial y}-\frac{\partial \overline{u^{\prime} v^{\prime}}}{\partial x}-\frac{\partial \overline{v^{\prime 2}}}{\partial y}
\end{gathered}
$$

and the conservation of tracer concentration

$$
\bar{u} \frac{\partial \bar{T}}{\partial x}+\bar{v} \frac{\partial \bar{T}}{\partial y}=-\frac{\partial \overline{u^{\prime} T^{\prime}}}{\partial x}-\frac{\partial \bar{v}^{\prime} T^{\prime}}{\partial y}
$$

Here $u^{\prime}$ and $v^{\prime}$ are the deviations in velocity from the time-averaged mean values $\bar{u}$ and $\bar{v}$ respectively, $\bar{p}$ is the pressure difference from the hydrostatic ambient pressure, $g$ is the gravitational acceleration, $\bar{T}$ is the time-averaged tracer concentration and $T^{\prime}$ the deviation from this mean value. An equation must also be provided for the relation between the density and the tracer concentration. The form

$$
\left(\bar{\rho}(x, y)-\rho_{a}\right) / \rho_{a}=\gamma \bar{T}(x, y)
$$

is assumed, where $\bar{T}(x, y)$ is the mean tracer concentration at any point $(x, y)$ in the jet and $\gamma$ may be a function of $\bar{T}(x, y)$. It is usual, however, to assume that $\gamma$ is a constant, although this approximation is not always adequate. The system of five equations (2.1)-(2.5) contains the eleven unknowns $\bar{u}, \bar{v}, \overline{u^{\prime 2}}, \bar{T}, \overline{T^{\prime 2}}, \overline{v^{\prime 2}}$, $\bar{p}, \overline{u^{\prime} T^{\prime}}, \overline{v^{\prime} T^{\prime}}, \bar{\rho}$ and $\overline{u^{\prime} v^{\prime}}$, and therefore could not be solved even if the appropriate boundary conditions were provided.

A large number of methods of dealing with this general problem have been proposed but the existing work on two-dimensional free shear flows (jets, plumes, wakes, etc.) can be classified into one of two schools of thought. The first tries to derive a constitutive equation, i.e. a relation (not necessarily linear) between the Reynolds-stress tensor and the deformation tensor of the fluid. The early workers in this school assumed, in addition, self-preservation for the mean and fluctuating quantities (see, for example, Tollmien 1926; Taylor 1932; or the textbook by Schlichting 1960, pp. 590-613). More recent workers use more complex constitutive equations and solve the appropriate system of equations numerically (see, for example, Lumley \& Khajeh-Nouri 1973; Saffman 1970; Cormack 1975). The second school integrates the equations of motion across the jet and derives ordinary differential equations for conservation integrals describing the mass, momentum and buoyancy fluxes. Corrsin \& Uberoi (1950), Townsend (1956) and Morton et al. (1956) have all used this approach. However, there are two major inherent difficulties with this technique. First, it is not at all clear where the limits of the integration across the jet should lie, and second, two unknown quantities are produced by the integration process. Most previous investigators have assumed that the limits of the spanwise integration can be taken at infinity, but as Crow \& Champagne (1971) pointed out for the case of an axially symmetric jet, this approach has problems in that certain of the integrals, e.g. the mass flux, are not strictly convergent. We choose limits of integration $\pm B(x)$ as the 
loci of points along which the mean forward velocity of particles travelling on the mean streamlines is zero. Such loci] do exist as shown by Reichardt (1942), Stewart (1956) and Taylor (1958). Although this definition does not guarantee that turbulent contributions are zero at the limit of integration, Miller \& Comings (1957) and Bradbury (1965) have shown that boundary contributions arising from these terms are small when considered in conjunction with the pressure field. With these assumptions the equations of motion reduce to

$$
\begin{gathered}
d \mu(x) / d x=-2 \bar{v}(x, B(x)), \\
\frac{d m(x)}{d x}=-\int_{-B(x)}^{B(x)} g \frac{\left(\bar{p}(x, y)-\rho_{a}\right)}{\rho_{a}} d y \\
d \beta(x) / d x=0
\end{gathered}
$$

where

$$
\begin{gathered}
\mu(x)=\int_{-B(x)}^{B(x)} \bar{u}(x, y) d y, \quad m(x)=\int_{-B(x)}^{B(x)} \bar{u}^{2}(x, y) d y, \\
\beta(x)=\gamma \int_{-B(x)}^{B(x)} g \frac{\frac{\left(\rho(x, y)-\rho_{a}\right) u(x, y)}{\rho_{a}}}{2} d y
\end{gathered}
$$

are the fluxes of specific mass, momentum and buoyancy respectively. Equation (2.8) is valid only if $\gamma$ is constant in the equation of state (2.5) for it is actually the tracer flux

that is conserved.

$$
\phi(x)=\int_{-B(x)}^{B(x)} \overline{u T} d y
$$

The fundamental problem in the integration of these equations is the description of the terms on the right-hand side. Several assumptions have generally been made in order to accomplish this.

Following Morton et al. (1956), Brooks \& Koh (1965) assumed that

$$
\lim _{B(x) \rightarrow \infty}-2 \bar{v}(x, B(x))=2 \alpha \bar{u}_{m}(x)
$$

i.e. they assumed that the entrainment velocity was directly proportional to the velocity on the axis of the jet. The entrainment coefficient $\alpha$ was assumed by Morton (1959) to be a universal constant with the same value for jets and plumes. The second assumption, on the basis of experimental evidence, has been to define the lateral distributions of velocity and tracer concentration to have exponential or other forms. Convenient distributions are

$$
\begin{aligned}
& \bar{u}(x, y)=\bar{u}_{m}(x) \exp \left[-\ln 2\left(y / b_{u}\right)^{2}\right], \\
& \bar{T}(x, y)=\bar{T}_{m}(x) \exp \left[-\ln 2\left(y / b_{T}\right)^{2}\right],
\end{aligned}
$$

where $b_{u}$ and $b_{T}$ are the characteristic lengths of the velocity and tracer profiles respectively and

$$
\bar{u}_{m}(x)=\bar{u}(x, 0), \quad \bar{T}_{m}(x)=\bar{T}(x, 0) .
$$

Further assumptions have been (i) that

$$
b_{T}(x) / b_{u}(x)=\lambda
$$


is a universal constant, (ii) that $\overline{u^{\prime} T^{\prime}} \ll \bar{u} . \bar{T}$, i.e. the turbulent flux of tracer is much less than the advective flux (Corrsin \& Uberoi 1950), and (iii) that the coefficient $\gamma$ relating density variation and tracer concentration is a constant.

Finally, it has been almost universally assumed that $B(x)$ can be taken as infinite without creating any problems. But, as we noted above, this is not without difficulties since it ignores the flow induced outside the jet by the jet itself. So far as the analysis of experimental results is concerned it is probably a fair assumption, since in practice the volume flux of a jet can be calculated to within experimental error by fitting a profile such as that defined by (2.11) and integrating to infinity. However, in the consideration of theoretical models that include both inner and outer flows it must be recognized that the integrals cannot extend to infinity. The choice of $B(x)$ given above is consistent with this.

The intention of the experiments to be reported here was to review the validity of the three basic assumptions enumerated above.

It will be shown that there is little apparent justification for their use, for under these assumptions the system of equations (2.5)-(2.8) becomes

$$
\begin{gathered}
d\left(\bar{u}_{m} b_{u}\right) / d x=2 \alpha \bar{u}_{m}\left(\pi^{-1} \ln 2\right)^{\frac{1}{2}} \\
d\left(\bar{u}_{m}^{2} b_{u}\right) / d x=2^{\frac{1}{2}} g \gamma \lambda \bar{T}_{m} b_{u}, \\
\frac{d}{d x}\left(\frac{\bar{T}_{m} \bar{u}_{m} b_{u}}{\left(1+\lambda^{2}\right)^{\frac{1}{2}}}\right)=0 .
\end{gathered}
$$

For a pure jet $\bar{\rho}(x, y)=\rho_{a}$, so that $\bar{T} \equiv 0$, and the specific momentum flux is preserved to within the approximations made. It is then easy to deduce from the above that

so that

$$
\left(d b_{u} / d x\right)_{\text {jet }}=4\left(\pi^{-1} \ln 2\right)^{\frac{1}{2}} \alpha,
$$

$$
\bar{u}_{m}(x) \sim m_{0}^{\frac{1}{2}} x^{-\frac{1}{2}}, \quad\left(\bar{T}_{m}(x)\right)_{\text {jet }} \sim x^{-\frac{1}{2}},
$$

where $m_{0}$ is the initial specific momentum flux and $\bar{T}_{m}(x)$ is interpreted as a mean tracer concentration, with no density contribution.

For a plume, the only dimensional parameter is the initial specific flux $\beta_{0}$. Thus dimensional analysis requires that

$$
\bar{u}_{m}(x)=\sigma_{u} \beta_{0}^{\mathrm{b}}, \quad\left(\bar{T}_{m}(x)\right)_{\mathrm{plume}} \sim x^{-1},
$$

for some constant $\sigma_{u}$, so that we find

$$
\left(d b_{u} / d x\right)_{\text {plume }}=2\left(\pi^{-1} \ln 2\right)^{\frac{1}{2}} \alpha .
$$

Now, if $\alpha$ is the same in both (2.18) and (2.21), then the rate of increase in width of a jet must be exactly twice that for a plume. This result was also found by Morton (1959) although he apparently did not consider it significant. It will be shown later that experimental measurements of $d b_{u} / d x$ for jets and plumes indicate that this is not so. We conclude that a constant entrainment coefficient is not adequate.

\section{Buoyant jets}

In view of the above our approach is to return to the basic approximate equations (2.6)-(2.8) and attempt to find an alternative description of the two unknown functions on the right-hand sides of these equations. In the absence of any well- 
defined turbulence model that enables the lateral distributions of velocity and temperature to be calculated, it seems appropriate to try and use dimensional analysis to gain some insight into the form of these functions. We use a method proposed by List \& Imberger (1973, 1975).

The entrainment function can be supposed to be a function of the variables $\mu, m, \beta$ and $x-x_{0}$, where $x_{0}$ is the virtual origin of the motion and is chosen such that the half-width of the jet there would be zero. Then we can write

$$
-2 \bar{v}(x, B(x))=(m / \mu) q(R, C),
$$

where $q$ is some as yet undetermined function of the two local dimensionless parameters $R$ and $C$, which are defined by

$$
R=\mu^{3} \beta / m^{3}, \quad C=\mu /\left[m\left(x-x_{0}\right)\right]^{\frac{1}{2}} .
$$

Since $m / \mu$ is the local velocity scale this result is consistent with Taylor's hypothesis that the entrainment is proportional to the local velocity scale. For a pure jet, $R$ is obviously zero, but for such a simple jet, where specific momentum is conserved, $C$ must be constant with a value $C_{j}$ say, so that $q\left(0, C_{j}\right)=\frac{1}{2} C_{j}^{2}$ (see List \& Imberger 1973). For a plume or buoyancy-driven flow it is also easy to show from dimensional analysis that $C$ and $R$ must be constants $\left(C_{p}\right.$ and $R_{p}$ respectively).

The buoyancy function on the right-hand side of (2.7) must also be a function of the same four variables $\mu, m, \beta$ and $x-x_{0}$, so that linearity in the gravitational acceleration implies that

$$
-\int_{-B(x)}^{B(x)} g \frac{\bar{\rho}(x, y)-\rho_{a}}{\rho_{a}} d y=\frac{\mu \beta}{m} h(R, C) .
$$

The functional forms of $q(R, C)$ and $h(R, C)$ are unknown, of course, and could only be completely determined from an integration of the complete equations of motion, which is impossible. However we note that, using the results above, (2.6) and (2.7) can be rewritten as

$$
\begin{gathered}
x \frac{d R}{d x}=\frac{3 R}{C^{2}}(q-R h), \\
x \frac{d C}{d x}=\frac{1}{C}\left(q-\frac{C^{2}}{2}-\frac{R h}{2}\right),
\end{gathered}
$$

provided that the buoyancy flux $\beta(x)$ is conserved. Since $x$ may be eliminated from these equations entirely, it is apparent that, if $q$ and $h$ were known, $C$ could be specified as a function of $R$ and some initial values $R_{0}$ and $C_{0}$. If the initial values were those corresponding to a pure jet, i.e. $R=0$ and $C=C_{j}$, then $q\left(0, C_{j}\right)=\frac{1}{2} C_{j}^{2}$. If the initial values were those corresponding to a pure plume, i.e. $R=R_{p}$ and $C=C_{p}$, then (2.26) and (2.27) imply that $q\left(R_{p}, C_{p}\right)=C_{p}^{2}$ and $h\left(R_{p}, C_{p}\right)=C_{p}^{2} / R_{p}$. We can therefore derive approximations for $q(R, C)$ and $h(R, C)$ either by direct linear interpolation or by expanding in Taylor series about the point $(R=0$, $C=C_{j}$ ). The two approximations are

$$
\begin{gathered}
q(R, C)=C_{p}^{2}(k+(1-k) \bar{R})+\ldots \\
h(R, C)=C_{\nu}^{2} / R_{p}+\ldots
\end{gathered}
$$


where $k=\frac{1}{2} C_{j}^{2} / C_{p}^{2}$ for the linear interpolation and $k=\frac{1}{2}$ for the Taylor series. Both of these results are of the same general form as that found by Priestley \& Ball (1955) for a round buoyant jet. With these expansions for $q$ and $h$ it is possible to solve (2.6) and (2.7). The only explicit solutions for general $k$ involve incomplete beta functions. However, for $k=\frac{1}{2}$ it can easily be shown that, in terms of the normalized variables

$$
\left.\begin{array}{c}
\xi=\left(x-x_{0}\right) C_{p}^{2}\left(\beta_{0} R_{p}^{-1}\right)^{\frac{2}{3}} / m_{0}, \quad \bar{m}=m / m_{0}, \\
\bar{\mu}=\mu\left(\beta_{0} R_{p}^{-1}\right)^{\frac{1}{3}} / m_{0}, \quad \bar{\beta}=\beta / \beta_{0}, \quad \bar{R}=R / R_{p}
\end{array}\right\}
$$

and with initial conditions

the solutions are

$$
\bar{\mu}=\left(R_{0} / R_{p}\right)^{\frac{1}{3}}=\bar{R}_{0}^{\frac{1}{3}}, \quad \bar{m}=1,
$$

and

$$
\bar{m}=\left[1-\bar{R}_{0}+\left(\xi+\bar{R}_{0}^{\frac{2}{3}}\right)^{\frac{3}{2}}\right]^{\frac{2}{3}},
$$

so that

$$
\bar{\mu}=\left(\xi+\bar{R}_{0}^{2}\right)^{\frac{1}{2}}\left[1-\bar{R}_{0}+\left(\xi+\bar{R}_{0}^{\frac{2}{2}}\right)^{\frac{3}{2}}\right]^{\frac{1}{3}}
$$

$$
\bar{R}=\frac{\left(\xi+\bar{R}_{0}^{\frac{2}{3}}\right)^{\frac{3}{2}}}{1-\bar{R}_{0}+\left(\xi+\bar{R}_{0}^{\frac{2}{3}}\right)^{\frac{3}{2}}} .
$$

The local velocity scale in a jet is specified by the ratio of the local specific momentum flux to the local volume flux, so that we can write

$$
\bar{u}_{m}(x)=a m / \mu,
$$

for some constant $a$. This equation becomes in dimensionless terms

$$
\frac{\bar{u}_{m}(x)}{\bar{u}_{0}}\left(\frac{R_{p}}{R_{0}}\right)^{\frac{1}{3}}=\frac{a\left[1-\bar{R}_{0}+\left(\xi+\bar{R}_{0}^{\frac{2}{3}}\right)^{\frac{3}{2}}\right]^{\frac{1}{3}}}{\left(\xi+\bar{R}_{0}^{\frac{g}{3}}\right)^{\frac{1}{2}}} .
$$

The asymptotic solutions for $\xi \gg 1$, i.e. plume-like flows, are easily seen to be

$$
\bar{m}=\xi, \quad \bar{\mu}=\xi, \quad \bar{R}=1, \quad \frac{\bar{u}_{m}(x)}{\bar{u}_{0}}\left(\frac{R_{p}}{R_{0}}\right)^{\frac{1}{3}}=a .
$$

When $\xi / \bar{R}_{0}^{s} \gg 1$, and in addition $\xi \ll 1$, then the solutions reduce to the forms appropriate for a pure jet:

$$
\bar{m}=1, \quad \bar{\mu}=\xi^{\frac{1}{2}}, \quad \bar{R}=\xi^{\frac{3}{2}}, \quad \frac{\bar{u}_{m}(x)}{\bar{u}_{0}}\left(\frac{R_{p}}{R_{0}}\right)^{\frac{1}{3}}=a \xi^{-\frac{1}{2}} .
$$

A comparison of the results predicted by these equations with experimentally determined values of the variables will be given in $\$ 4$. It should be noted however that the solutions given will only be valid for $\xi>\bar{R}_{0}^{3}$, since it may be shown that when $\xi=\bar{R}_{0}^{2}, x-x_{0}$ is of the order of 2.5 jet widths, which is really too close to expect fully developed jet and plume solutions to be applicable.

Before discussing the experiments that have been performed to evaluate this approach it is worthwhile to reconsider (2.9). If we define a local mean value of $\gamma$ by $\bar{\gamma}$ then we can write

$$
\beta(x)=g \bar{\gamma} \int_{-B(x)}^{B(x)} \overline{u(x, y) T(x, y)} d y
$$


Now, recognizing that there is no basic reason for lack of self-similarity in either pure jets or pure plumes, we should expect that the relative flux of tracer carried by advection and turbulence should remain constant in both a pure jet and a pure plume. Thus we should expect that

$$
\int_{-B(x)}^{B(x)} \overline{u(x, y) T(x, y)} d y \sim \bar{u}_{m}(x) \bar{T}_{m}(x)\left(x-x_{0}\right)
$$

with a different proportionality constant in each case. Using the similarity results (2.19) and (2.20) and noting that for jet-like flows $\beta(x)=\beta_{0}$ we should expect

$$
\sigma_{T}=\frac{\beta^{\frac{2}{3}}}{g \bar{\gamma}\left(x-x_{0}\right) \bar{T}_{m}(x)}
$$

to be a constant if $x-x_{0} \geqslant m_{0} / \beta_{0}^{\frac{2}{3}}$ and to be proportional to $\left[\left(x-x_{0}\right) \beta_{0}^{\frac{2}{3}} / m_{0}\right]^{-\frac{1}{2}}$ for $\left(x-x_{0}\right) \beta_{0}^{\frac{3}{3}} / m_{0} \ll 1$. We shall use the experimental results to confirm these arguments.

\section{Experiments}

The objective of the experimental investigation was to study the mechanics of a plane vertical turbulent jet for a wide range of initial Richardson numbers. Velocity, temperature and heat flux were measured and their time-averaged values and root-mean-square deviations computed. In addition, conditional averaging of the velocity and temperature signals was performed. However, in this paper we shall only report on the time-averaged results; the turbulent variations will be the subject of part 2 .

The experiments took three basic forms: experiments in which only temperature measurements were taken, experiments in which only velocities were recorded and experiments in which velocities and temperatures were recorded simultaneously. The last set of experiments resulted in computations of the timeaveraged heat flux within the turbulent jet.

Before discussing the results of the experiments some description of the experimental procedures and techniques is in order. A two-dimensional vertical buoyant jet was generated by discharging heated water from a chamber containing flowstraightening elements through a slot $13 \mathrm{~cm}$ long whose width could be varied from $2 \mathrm{~mm}$ to $10 \mathrm{~mm}$ (see figure 1). The jet discharged into a tank $4 \mathrm{~m}$ square and $1 \mathrm{~m}$ deep filled with water of uniform temperature. The jet was confined by two Plexiglas walls $13 \mathrm{~cm}$ apart in order to maintain two-dimensionality of the flow. A constant-head source supplied the jet through a calibrated flowmeter. In all the experiments the tracer concentration measured was the excess temperature of the jet fluid above the ambient fluid.

Six small bead thermistors were used for temperature measurements. The bead diameter was $0.3 \mathrm{~mm}$ and the dissipation constant in still water at $25^{\circ} \mathrm{C}$ was 0.75 $\mathrm{mW} /{ }^{\circ} \mathrm{C}$. The self-heating of the thermistor due to Ohmic dissipation, calculated using the dissipation constant, was less than $0.008^{\circ} \mathrm{C}$. The time constant for a $63 \%$ response was found to be $27 \mathrm{~ms}$ for one thermistor with special insulation and $45 \mathrm{~ms}$ for the five others. 


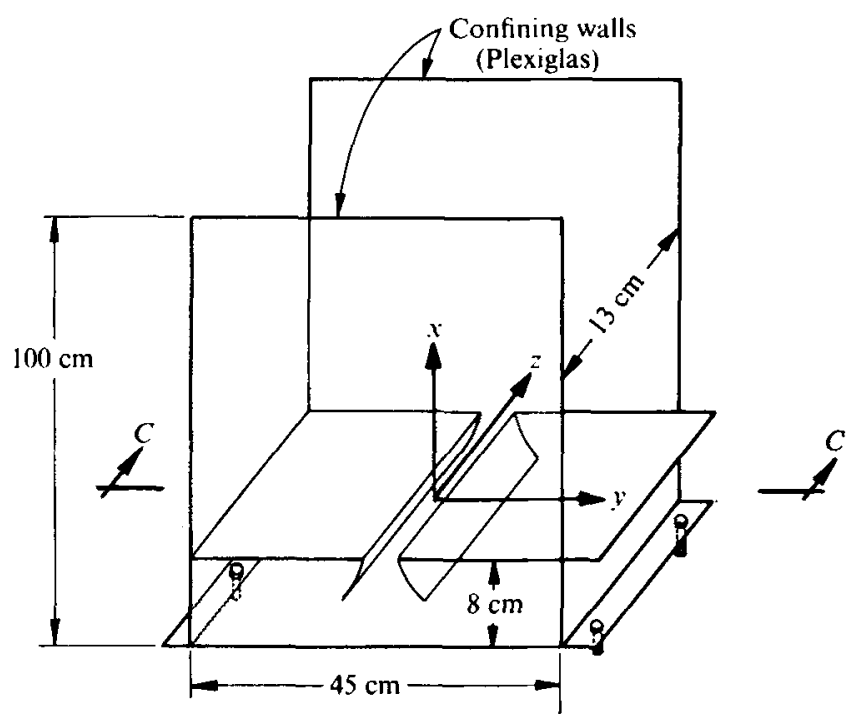

(a)

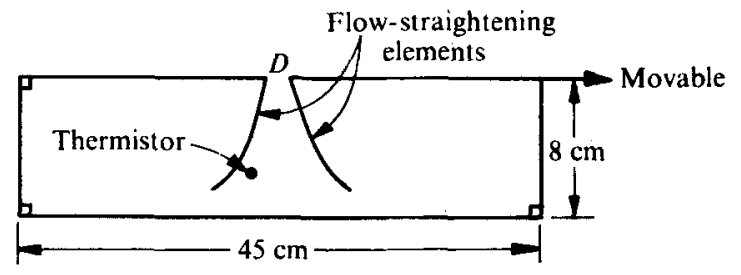

(b)

Figure 1. (a) Perspective view and (b) cross-section of the jet chamber.

Each bead thermistor was insulated and mounted on a stainless-steel tube to form a temperature probe that could be located at any desired site within the buoyant jet. The thermistor temperature response was measured with a bridge circuit; each thermistor was individually calibrated and a third-order polynomial fitted to the set of calibration points in the least-squares sense. The drift of the thermistor circuit for an extended period of time $(3 \mathrm{~h})$ was of the order of $1 \mathrm{mV}$, resulting in an absolute accuracy of the temperature measurement of the order of $0.01{ }^{\circ} \mathrm{C}$.

Each analog output corresponding to temperature was fed into one channel of an eight-channel analog-to-digital data acquisition and recording system. The digitizing resolution was $1 \mathrm{mV}$ and the sampling rate could be varied from 0.01 to 1600 samples/s.

An investigation was performed to determine the lowest sampling rate and the smallest sample time that would give a time-averaged mean temperature at any location within the jet, with a standard deviation of $\pm 0.02{ }^{\circ} \mathrm{C}$ from the ideal. These tests were performed by first forming a file of data at $400 \mathrm{samples} / \mathrm{s}$ and 
then manipulating these data on a digital computer to determine the appropriate sampling rate and sample time; $10 \mathrm{samples} / \mathrm{s}$ and a sample time of $150 \mathrm{~s}$ were found to be adequate, indicating a high frequency cut-off at about $5 \mathrm{~Hz}$.

The vertical velocity in the jet was measured using a forward-scattering/ reference-beam laser-Doppler velocimeter. The beam from a $5 \mathrm{~mW}$ helium-neon laser was first passed through a rotating radial diffraction-grating disk mounted on the shaft of a synchronous motor rotating at 1800 r.p.m. The disk contained 2048 lines. The zeroth-order diffracted laser light then had the frequency $f_{0}$ of the laser beam, and the first-order diffracted beams had a frequency $f_{1}=f_{0} \pm 61 \cdot 440$ $\mathrm{kHz}$ (Stevenson 1970). The zeroth-order (frequency $f_{0}$ ) and first-order (frequency $f_{1}$ ) diffracted light beams were then passed through a beam splitter in order to increase their separation; all other diffracted light was masked. A lens focused the two beams of frequencies $f_{0}$ and $f_{1}$ into the flow field and a photomultiplier tube was aligned with the beam of frequency $f_{1}$ (the 'reference beam') after it had passed through the flow. The photomultiplier also collected light of frequency $f_{s}$ that was scattered by particles (of diameter of the order of $1 \mu \mathrm{m}$ ) existing in abundance in the flow field. The heterodyning of the scattered light with the reference beam formed the basis of the velocity measurement system.

The output from the photomultiplier tube was then passed to a frequency spectrum analyser. The output signal spectrum so produced can then be related to the probability distribution of velocity at the focus point of the two laser beams since there is a linear relationship between the frequency of the heterodyne signal and the velocity of particles passing through the focal volume. There is, as is well known, possible signal spectrum broadening resulting from noise within the laser, velocity shears within the scattering volume, refractive-index fluctuations and so forth. The result of such broadening is to increase the apparent turbulence intensity by increasing the variance of the signal spectrum. Some measure of correction for these errors was made by subtracting the zero velocity signal spectrum from the spectrum determined at each point in the fluid. This is not rigorously correct, since it is not possible to subtract spectra from one another because of the convolution property of transfer functions. However, the mean velocity at the jet exit determined from the first moment of the probability distribution of velocity so adjusted agreed to within better than $1 \%$ with the velocity determined on the basis of the mass efflux rate.

The amplitude of the spectrum and the corresponding frequency were both digitized and stored on magnetic tape for subsequent analysis. A reasonably smooth probability density function of velocity was obtained with a sample rate of $40 \mathrm{samples} / \mathrm{s}$ and a sample time of $10 \mathrm{~min}$.

The two-dimensionality of the flow field was carefully verified and details of these studies may be found in Kotsovinos (1975).

\section{Experimental results}

A substantial number of experiments on plane buoyant jets were performed using the apparatus and techniques described in the previous section. In these experiments, mean velocity, mean temperature and buoyancy flux profiles were 


\begin{tabular}{|c|c|c|c|c|c|c|c|}
\hline Experiment & $R_{0}$ & $\begin{array}{c}D \\
(\mathrm{~cm})\end{array}$ & $\begin{array}{c}x \\
(\mathrm{~cm})\end{array}$ & $\begin{array}{c}\bar{T}_{\mathbf{0}} \\
\left({ }^{\circ} \mathbf{C}\right)\end{array}$ & $\begin{array}{l}\bar{T}_{m} \\
\left({ }^{\circ} \mathrm{C}\right)\end{array}$ & $\begin{array}{c}b_{T} \\
(\mathrm{~cm})\end{array}$ & $\sigma_{T}$ \\
\hline PLVT2-E & 0.000051 & 0.24 & $5 \cdot 0$ & $3 \cdot 45$ & $1 \cdot 75$ & 0.79 & $2 \cdot 660$ \\
\hline PLVT2-Z & 0.000089 & 0.24 & $8 \cdot 9$ & $5 \cdot 40$ & $2 \cdot 02$ & $1 \cdot 40$ & $1 \cdot 722$ \\
\hline PLVT2-H & 0.000058 & $0 \cdot 24$ & $14 \cdot 1$ & $3 \cdot 80$ & $1 \cdot 16$ & $2 \cdot 04$ & $1 \cdot 501$ \\
\hline PLVT2-A & 0.000123 & 0.24 & $22 \cdot 5$ & $6 \cdot 85$ & $1 \cdot 80$ & $3 \cdot 30$ & 0.858 \\
\hline JUNE-3 & $0 \cdot 00091$ & 0.5 & $5 \cdot 0$ & $11 \cdot 20$ & $7 \cdot 10$ & 0.90 & $1 \cdot 157$ \\
\hline JUNE-3 & 0.00091 & 0.5 & $10 \cdot 0$ & $11 \cdot 20$ & $5 \cdot 15$ & $1 \cdot 53$ & 0.961 \\
\hline JUNE-3 & 0.00091 & 0.5 & $15 \cdot 0$ & $11 \cdot 20$ & $4 \cdot 00$ & $2 \cdot 21$ & $0 \cdot 889$ \\
\hline JUNE-3 & 0.00091 & 0.5 & $25 \cdot 0$ & $11 \cdot 20$ & $3 \cdot 10$ & $3 \cdot 50$ & 0.734 \\
\hline JUNE-7 & 0.00094 & 0.5 & $5 \cdot 0$ & $6 \cdot 60$ & $4 \cdot 25$ & $0 \cdot 80$ & $1 \cdot 174$ \\
\hline JUNE-7 & 0.00094 & 0.5 & $10 \cdot 0$ & $6 \cdot 60$ & $3 \cdot 20$ & $1 \cdot 50$ & 0.911 \\
\hline JUNE-7 & 0.00094 & 0.5 & $15 \cdot 2$ & $6 \cdot 60$ & $2 \cdot 50$ & $2 \cdot 20$ & 0.818 \\
\hline JUNE-7 & $0 \cdot 00094$ & 0.5 & $25 \cdot 5$ & $6 \cdot 60$ & $1 \cdot 80$ & $3 \cdot 62$ & $0 \cdot 714$ \\
\hline JUNE-7 & 0.00094 & 0.5 & $30 \cdot 0$ & $6 \cdot 60$ & $1 \cdot 74$ & $4 \cdot 20$ & $0 \cdot 635$ \\
\hline PLVT3-B & 0.00189 & $0 \cdot 24$ & $8 \cdot 1$ & $9 \cdot 20$ & $3 \cdot 05$ & $1 \cdot 16$ & 0.800 \\
\hline PLVT3-A & 0.00224 & $0 \cdot 24$ & $22 \cdot 5$ & $10 \cdot 40$ & $1 \cdot 78$ & $3 \cdot 10$ & 0.518 \\
\hline JULY-3 & 0.0058 & $1 \cdot 0$ & $6 \cdot 0$ & $5 \cdot 10$ & $3 \cdot 68$ & $1 \cdot 28$ & 0.705 \\
\hline JULY-3 & $0 \cdot 0058$ & $1 \cdot 0$ & $14 \cdot 0$ & $5 \cdot 10$ & $2 \cdot 43$ & $2 \cdot 30$ & 0.625 \\
\hline JULY - 3 & 0.0058 & $1 \cdot 0$ & $22 \cdot 0$ & $5 \cdot 10$ & 1.76 & $3 \cdot 20$ & 0.612 \\
\hline JULY-3 & 0.0058 & $1 \cdot 0$ & $30 \cdot 4$ & $5 \cdot 10$ & $1 \cdot 56$ & $4 \cdot 20$ & 0.527 \\
\hline JULY-3 & 0.0058 & 1.0 & $36 \cdot 0$ & $5 \cdot 10$ & $1 \cdot 43$ & $4 \cdot 80$ & 0.497 \\
\hline JULY -5 & 0.0050 & $1 \cdot 0$ & $6 \cdot 0$ & $4 \cdot 50$ & $3 \cdot 00$ & $1 \cdot 27$ & 0.838 \\
\hline JULY-5 & 0.0050 & $1 \cdot 0$ & $14 \cdot 0$ & $4 \cdot 50$ & $2 \cdot 15$ & $2 \cdot 20$ & 0.669 \\
\hline JULY-5 & 0.0050 & $1 \cdot 0$ & $22 \cdot 0$ & $4 \cdot 50$ & $1 \cdot 56$ & $3 \cdot 33$ & 0.649 \\
\hline JULY-5 & $0 \cdot 0050$ & $1 \cdot 0$ & $30 \cdot 4$ & $4 \cdot 50$ & $1 \cdot 37$ & $4 \cdot 20$ & 0.562 \\
\hline JULY-5 & $0 \cdot 0050$ & $1 \cdot 0$ & $36 \cdot 2$ & $4 \cdot 50$ & $1 \cdot 25$ & $4 \cdot 90$ & 0.529 \\
\hline JULY-25 & $0 \cdot 030$ & $1 \cdot 0$ & $6 \cdot 0$ & $18 \cdot 30$ & $12 \cdot 00$ & $1 \cdot 13$ & 0.607 \\
\hline JULY-25 & $0 \cdot 030$ & $1 \cdot 0$ & $14 \cdot 0$ & $18 \cdot 30$ & $7 \cdot 25$ & $2 \cdot 00$ & 0.532 \\
\hline JULY-25 & 0.030 & $1 \cdot 0$ & $22 \cdot 0$ & $18 \cdot 30$ & $5 \cdot 25$ & $3 \cdot 00$ & 0.502 \\
\hline JULY-25 & $0 \cdot 030$ & $1 \cdot 0$ & $20 \cdot 0$ & $18 \cdot 30$ & $4 \cdot 15$ & 4.05 & 0.483 \\
\hline JULY-25 & 0.030 & $1 \cdot 0$ & $36 \cdot 0$ & $18 \cdot 30$ & $3 \cdot 55$ & $4 \cdot 70$ & 0.479 \\
\hline JUNE-6 & 0.030 & 0.5 & $5 \cdot 0$ & $23 \cdot 50$ & $12 \cdot 70$ & $0 \cdot 80$ & $0 \cdot 467$ \\
\hline JUNE-6 & 0.030 & 0.5 & $10 \cdot 0$ & $23 \cdot 50$ & $7 \cdot 80$ & $1 \cdot 43$ & 0.455 \\
\hline JUNE.6 & 0.030 & 0.5 & $15 \cdot 0$ & $23 \cdot 50$ & $5 \cdot 50$ & $2 \cdot 03$ & 0.463 \\
\hline JUNE-6 & 0.030 & 0.5 & $25 \cdot 0$ & $23 \cdot 50$ & $3 \cdot 75$ & $3 \cdot 22$ & 0.434 \\
\hline JUNE-6 & 0.030 & 0.5 & $30 \cdot 0$ & $23 \cdot 50$ & $3 \cdot 40$ & $3 \cdot 77$ & 0.405 \\
\hline JULY-10 & 0.063 & $1 \cdot 0$ & $6 \cdot 0$ & $15 \cdot 10$ & $8 \cdot 30$ & $1 \cdot 10$ & 0.509 \\
\hline JULY-10 & 0.063 & $1 \cdot 0$ & $14 \cdot 0$ & $15 \cdot 10$ & $5 \cdot 25$ & $2 \cdot 07$ & 0.445 \\
\hline JULY-10 & 0.063 & $1 \cdot 0$ & $22 \cdot 0$ & $15 \cdot 10$ & $3 \cdot 45$ & $3 \cdot 00$ & 0.473 \\
\hline JULY-10 & 0.063 & $1 \cdot 0$ & $30 \cdot 4$ & $15 \cdot 10$ & $3 \cdot 00$ & $3 \cdot 83$ & 0.410 \\
\hline JULY-10 & $0 \cdot 063$ & $1 \cdot 0$ & $36 \cdot 2$ & $15 \cdot 10$ & $2 \cdot 50$ & $4 \cdot 67$ & 0.422 \\
\hline PLVT1-F & $0 \cdot 178$ & $1 \cdot 0$ & $9 \cdot 0$ & $11 \cdot 50$ & $3 \cdot 87$ & $1 \cdot 70$ & 0.503 \\
\hline PLVT1-C & 0.179 & $1 \cdot 0$ & $14 \cdot 0$ & $11 \cdot 55$ & $3 \cdot 25$ & $2 \cdot 34$ & 0.416 \\
\hline PLVT1-Z & $0 \cdot 166$ & 1.0 & $19 \cdot 0$ & $11 \cdot 00$ & $2 \cdot 20$ & $2 \cdot 55$ & 0.458 \\
\hline PLVT1-C & $0 \cdot 168$ & $1 \cdot 0$ & $23 \cdot 5$ & $10 \cdot 90$ & $2 \cdot 00$ & $3 \cdot 25$ & 0.414 \\
\hline PLVT1-K & $0 \cdot 177$ & $1 \cdot 0$ & $28 \cdot 8$ & $11 \cdot 15$ & 1.53 & $3 \cdot 75$ & 0.452 \\
\hline PLVT1-B & $0 \cdot 170$ & $1 \cdot 0$ & $33 \cdot 6$ & $11 \cdot 00$ & $1 \cdot 35$ & $4 \cdot 40$ & 0.446 \\
\hline PLVT1-H & $0 \cdot 182$ & $1 \cdot 0$ & $39 \cdot 1$ & $11 \cdot 40$ & $1 \cdot 22$ & 4.95 & 0.433 \\
\hline JUNE-28 & $0 \cdot 216$ & $1 \cdot 0$ & $6 \cdot 0$ & $20 \cdot 90$ & $9 \cdot 60$ & $1 \cdot 07$ & $0 \cdot 447$ \\
\hline JUNE-28 & 0.216 & $1 \cdot 0$ & $14 \cdot 0$ & $20 \cdot 90$ & $5 \cdot 60$ & $1 \cdot 97$ & 0.413 \\
\hline JUNE-28 & $0 \cdot 216$ & $1 \cdot 0$ & $22 \cdot 0$ & $20 \cdot 90$ & $3 \cdot 50$ & $3 \cdot 00$ & 0.457 \\
\hline JUNE-28 & 0.216 & $1 \cdot 0$ & $30 \cdot 2$ & $20 \cdot 90$ & $2 \cdot 70$ & $4 \cdot 13$ & 0.449 \\
\hline JUNE-28 & 0.216 & $1 \cdot 0$ & $36 \cdot 2$ & $20 \cdot 90$ & $2 \cdot 30$ & $4 \cdot 80$ & $0 \cdot 448$ \\
\hline
\end{tabular}

TaBle 1 (continued). 


\begin{tabular}{|c|c|c|c|c|c|c|c|}
\hline Experiment & $\boldsymbol{R}_{0}$ & $\begin{array}{c}D \\
(\mathrm{~cm})\end{array}$ & $\begin{array}{c}x \\
(\mathrm{~cm})\end{array}$ & $\begin{array}{c}\bar{T}_{0} \\
\left({ }^{\circ} \mathrm{C}\right)\end{array}$ & $\begin{array}{c}\bar{T}_{m} \\
\left({ }^{\circ} \mathrm{C}\right)\end{array}$ & $\begin{array}{c}b_{T} \\
(\mathrm{~cm})\end{array}$ & $\sigma_{T}$ \\
\hline IULY -1 & $0 \cdot 231$ & $1 \cdot 0$ & $6 \cdot 0$ & $21 \cdot 85$ & $10 \cdot 20$ & 1.07 & 0.418 \\
\hline JULY -1 & $0 \cdot 231$ & $1 \cdot 0$ & $14 \cdot 0$ & $21 \cdot 85$ & $5 \cdot 65$ & $1 \cdot 97$ & $0 \cdot 414$ \\
\hline JULY -1 & $0 \cdot 231$ & $1 \cdot 0$ & $22 \cdot 0$ & $21 \cdot 85$ & $3 \cdot 70$ & $2 \cdot 87$ & 0.438 \\
\hline JULY - 1 & 0.231 & $1 \cdot 0$ & $30 \cdot 4$ & $21 \cdot 85$ & $2 \cdot 80$ & $4 \cdot 00$ & 0.438 \\
\hline JULY-1 & 0.231 & $1 \cdot 0$ & $36 \cdot 4$ & $21 \cdot 85$ & $2 \cdot 40$ & $4 \cdot 70$ & 0.435 \\
\hline JUNE-26 & $0 \cdot 295$ & $2 \cdot 0$ & $12 \cdot 0$ & $21 \cdot 90$ & $8 \cdot 50$ & $2 \cdot 26$ & $0 \cdot 419$ \\
\hline JUNE-26 & 0.295 & $2 \cdot 0$ & $20 \cdot 0$ & $21 \cdot 90$ & $6 \cdot 25$ & $3 \cdot 32$ & 0.417 \\
\hline JUNE-26 & 0.295 & $2 \cdot 0$ & $28 \cdot 0$ & $21 \cdot 90$ & $4 \cdot 80$ & $4 \cdot 00$ & 0.429 \\
\hline JUNE-26 & 0.295 & $2 \cdot 0$ & $36 \cdot 0$ & $21 \cdot 90$ & $4 \cdot 20$ & $5 \cdot 00$ & 0.404 \\
\hline JULY-29 & $0 \cdot 424$ & $1 \cdot 0$ & $6 \cdot 0$ & $21 \cdot 70$ & $9 \cdot 00$ & $1 \cdot 17$ & $0 \cdot 328$ \\
\hline JULY-29 & 0.424 & $1 \cdot 0$ & $14 \cdot 0$ & $21 \cdot 70$ & $4 \cdot 65$ & $2 \cdot 07$ & 0.374 \\
\hline JULY-29 & 0.424 & $1 \cdot 0$ & $22 \cdot 0$ & $21 \cdot 70$ & $3 \cdot 12$ & $2 \cdot 95$ & $0 \cdot 394$ \\
\hline JULY-29 & 0.424 & $1 \cdot 0$ & $30 \cdot 0$ & $21 \cdot 70$ & $2 \cdot 30$ & $3 \cdot 95$ & 0.414 \\
\hline JULY-29 & 0.424 & $1 \cdot 0$ & $36 \cdot 0$ & $21 \cdot 70$ & $2 \cdot 06$ & $4 \cdot 90$ & 0.395 \\
\hline
\end{tabular}

TABLE 1. Experimental values for temperature half-width and temperature distribution along jet axis for turbulent jets and plumes.

measured for jets with different initial Richardson numbers. A total of 102 velocity and temperature cross-sections were measured. Of these, 66 were temperature cross-sections and 36 velocity cross-sections. There were 13 simultaneous measurements of velocity and temperature cross-sections. The initial Richardson numbers of the jets varied from 0 to $0 \cdot 70$. The relevant experimental parameters for all the tests will be presented in this section along with a discussion of the results on mean temperature, mean velocity and mean buoyancy flux.

\section{Mean temperature profiles}

The time-averaged mean temperature $\bar{T}(x, y)$ for particular distances from the jet origin was plotted as a function of the transverse distance $y$ (in $\mathrm{cm}$ ) for each experiment at a fixed initial Richardson number. A Gaussian curve of the form (2.12) was then fitted to the experimental data and the temperature half-width as a function of distance determined. Detailed plots of each temperature profile are available in Kotsovinos (1975). The results for all 66 experiments in which the mean temperature profile was measured are given in table 1. The maximum temperature on the jet axis for all experiments is also given in table 1 and from these results it is possible to plot curves of the maximum temperature and jet half-width as a function of distance from the jet origin. The jet half-width $b_{T}$ is then seen to be a remarkably linear function of distance from the jet origin for each experiment performed. These linear growth curves for the half-width were used to define the virtual origin $x_{0}$ for each jet experiment plotted in figure 2 .

According to the arguments presented previously we should expect to find that in a buoyant jet

$$
\sigma_{T}=\frac{\beta^{\frac{2}{3}}}{g \bar{\gamma}\left(x-x_{0}\right) \bar{T}_{m}(x)}
$$

becomes asymptotic to a constant for $x-x_{0} \gg m_{0} / \beta_{0}^{3}$. For small values of $x-x_{0}$ we should expect $\sigma_{T}$ to be asymptotic to a line with slope $-\frac{1}{2}$. Figure 2 shows the 


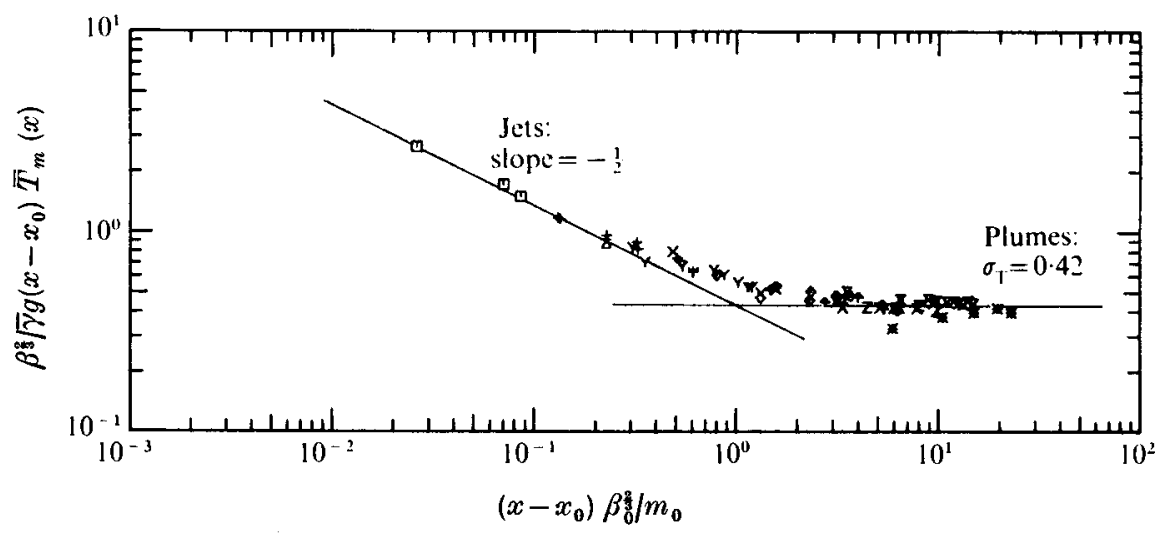

Figure 2. Dimensionless time-averaged temperature distribution on the axis of turbulent buoyant jets. Initial Richardson numbers $R_{0}: \square, 0.00006 ; \triangle, 0.00012 ;+, 0.0009 ; \times$, $0.002 ; \mathrm{Y}, 0.005 ; \diamond, 0.03 ; \triangle, 0.06 ; \bar{x}, 0.17,0 \cdot 22 ; \mathrm{Z}, 0.29 ; *, 0 \cdot 42$.

experimental results obtained from a series of experiments at different Richardson numbers plotted as a function of the normalized variable $\left(x-x_{0}\right) \beta_{0}^{2} / m_{0}$. The division between jets and plumes is particularly evident. The buoyancy flux used in these results is that obtained from the source scaled by the variation in the thermal coefficient of expansion $\bar{\gamma}$.

\section{Mean velocity profiles}

The time-averaged mean velocity $\bar{u}(x, y)$ was recorded in both jet and plume-like flows as a function of lateral displacement for several distances downstream from the jet origin. A Gaussian curve was fitted to the experimental data at each crosssection and the velocity half-width determined from an empirically fitted curve given by (2.11). The results are presented in table 2 , along with several other variables of interest that will be discussed subsequently. The space available precludes including graphs of the velocity profiles and half-widths but the data given in table 2 , when plotted, do show that the half-widths are linear functions of distance from the jet origin for each of the experiments performed.

Table 2 also includes values of the volume flux and specific momentum flux calculated from the Gaussian fitted profiles. In addition, the local values of the mean local buoyancy flux $\beta(x)$ are given.

With the values of the volume, momentum and buoyancy flux specified at a definite number of points along the jet it becomes possible to calculate the local spreading-rate coefficient $C$ and the local jet Richardson number $R$ at these points. These are also given in table 2.

Since the length scale separating plumes from jets is $m_{0} / \beta_{0}^{3}$, graphs of $C(x)$ and $R(x) v s .\left(x-x_{0}\right) \beta_{0}^{\frac{2}{3}} / m_{0}$ should indicate the values of these variables appropriate for jets and plumes. These graphs are given in figures $3(a)$ and $(b)$.

The values of $C$ given in figure $3(a)$ are those calculated at each point where a velocity profile was measured. The virtual origin used in each experiment was that obtained from a graph of the velocity half-width against distance from the 


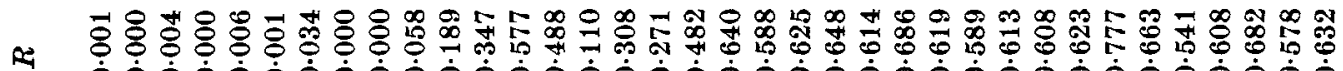
R $\dot{0} \dot{0} \dot{0} \dot{0} \dot{0} \dot{0} \dot{0} \dot{0} \dot{0} \dot{0} \dot{0} \dot{0} \dot{0} \dot{0} \dot{0} \dot{0} \dot{0} \dot{0} \dot{0} \dot{0} \dot{0} \dot{0} \dot{0} \dot{0} \dot{0} \dot{0} \dot{0} \dot{0} \dot{0} \dot{0} \dot{0} \dot{0} \dot{0}$

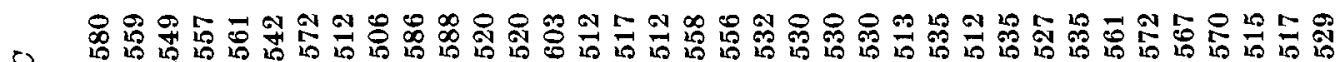

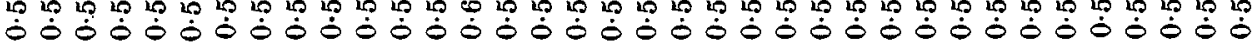

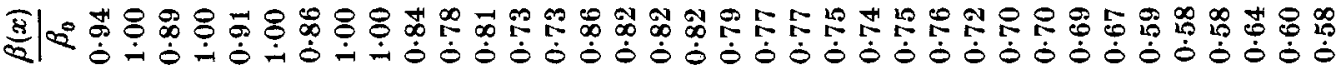

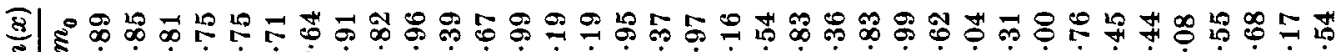
हl| है

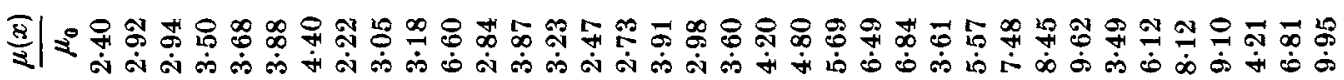

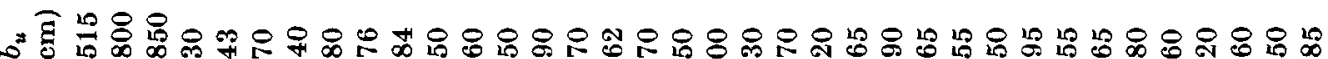

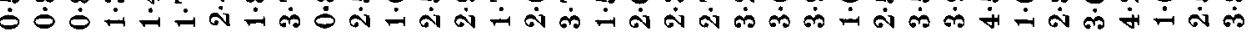

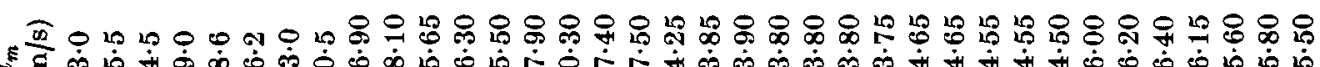

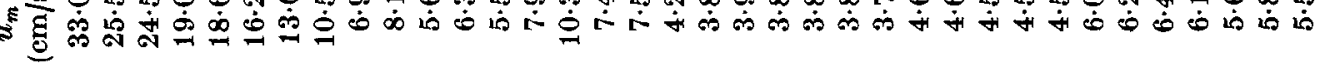

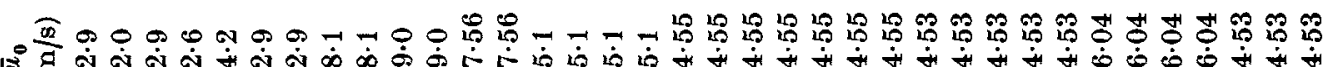
1 死

\& 大ิ

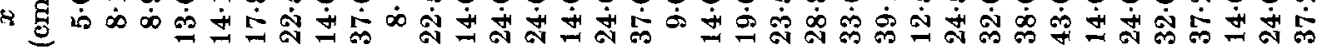

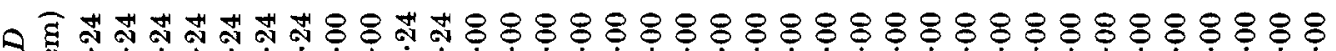

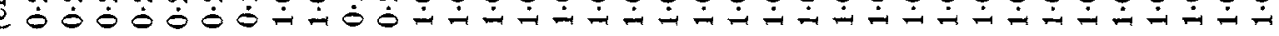

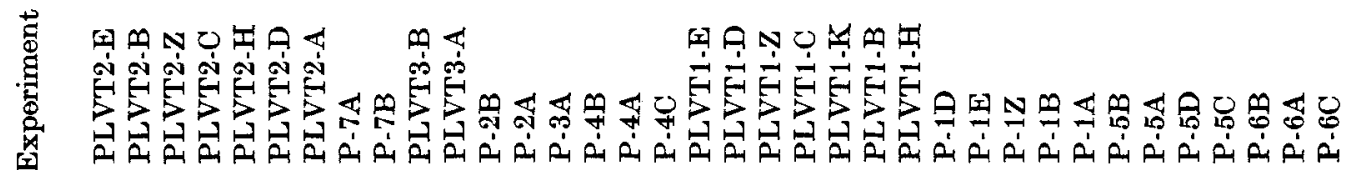

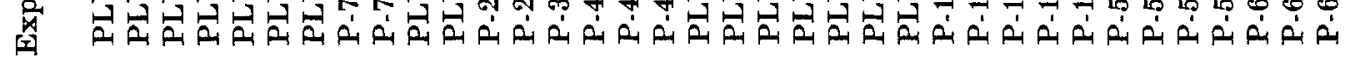



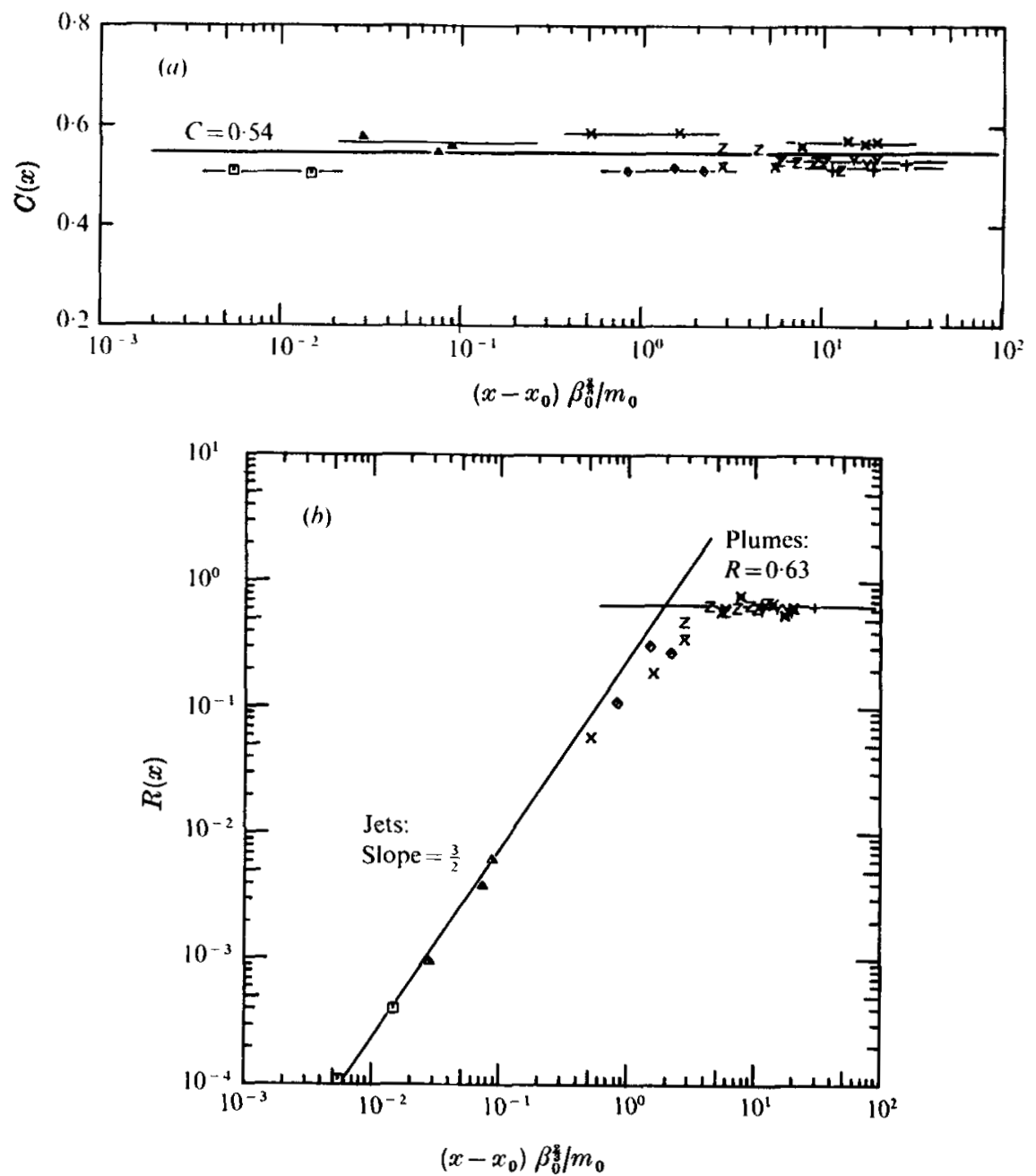

Figure 3. (a) Jet width parameter (2.24) and (b) local jet Richardson number (2.23) as a function of dimensionless distance from the jet origin. Initial Richardson numbers $R_{0}$ : $\square, 0.000008 ; \triangle, 0.00006 ; \times, 0.002 ; \diamond, 0.015 ; \bar{x}, 0.1 ; \mathrm{Z}, 0.17 ; \mathrm{Y}, 0.31 ; \otimes, 0.4 ;+, 0.7$.

actual jet origin. It can be seen that $C(x)$ is fairly constant for each experiment as one would expect, since for the Gaussian profiles used to fit the experimental data it may easily be shown that

$$
\frac{d b_{u}}{d x}=\left(\frac{\ln 2}{2 \pi}\right)^{\frac{1}{2}} C^{2}
$$

However, what is even more interesting is the fact that, although there is some scatter in the data, $C$ does appear to be relatively independent of whether the flow is jet-like or plume-like in character. A reasonable value for this constant, $C_{p}$, is 0.54 . The actual numerical values of $C$ and $x_{0} / D$, derived from the above relation and the plots of velocity half-width for each experiment, are given in table 3. 


\begin{tabular}{|c|c|c|c|c|c|c|}
\hline \multirow[t]{2}{*}{ Experiment } & \multirow[t]{2}{*}{$R_{0}$} & \multicolumn{2}{|c|}{$x / D$} & \multirow[t]{2}{*}{$d b_{u} / d x$} & \multirow[t]{2}{*}{$C$} & \multirow[t]{2}{*}{$+x_{0} / D$} \\
\hline & & From & To & & & \\
\hline P.7 & 0.0 & $14 \cdot 0$ & $37 \cdot 0$ & 0.087 & 0.512 & $-6 \cdot 7$ \\
\hline PLVT-2 & $\sim 0.0$ & $20 \cdot 8$ & $93 \cdot 7$ & $0 \cdot 109$ & 0.573 & +2.5 \\
\hline PLVT-3 & 0.002 & $33 \cdot 7$ & $94 \cdot 0$ & $0 \cdot 106$ & 0.565 & $+3 \cdot 1$ \\
\hline P-4 & 0.015 & $14 \cdot 0$ & $37 \cdot 0$ & 0.087 & 0.512 & $-5 \cdot 6$ \\
\hline P-2 & $0 \cdot 10$ & $14 \cdot 0$ & $24 \cdot 0$ & 0.09 & 0.521 & $-3 \cdot 8$ \\
\hline PLVT-1 & $0 \cdot 17$ & $9 \cdot 0$ & $39 \cdot 1$ & 0.093 & 0.529 & $-5 \cdot 87$ \\
\hline P-1 & 0.31 & $12 \cdot 5$ & $43 \cdot 0$ & 0.095 & 0.535 & -4.85 \\
\hline P-5 & 0.40 & $14 \cdot 0$ & $37 \cdot 2$ & $0 \cdot 104$ & 0.560 & $-1 \cdot 78$ \\
\hline P-6 & $0 \cdot 70$ & $14 \cdot 0$ & $37 \cdot 2$ & 0.093 & 0.529 & $-4 \cdot 20$ \\
\hline
\end{tabular}

TABLE 3. Values of half-width slope and virtual origin derived from velocity measurements. Mean value of growth coefficient $C$ is $0 \cdot 54$.

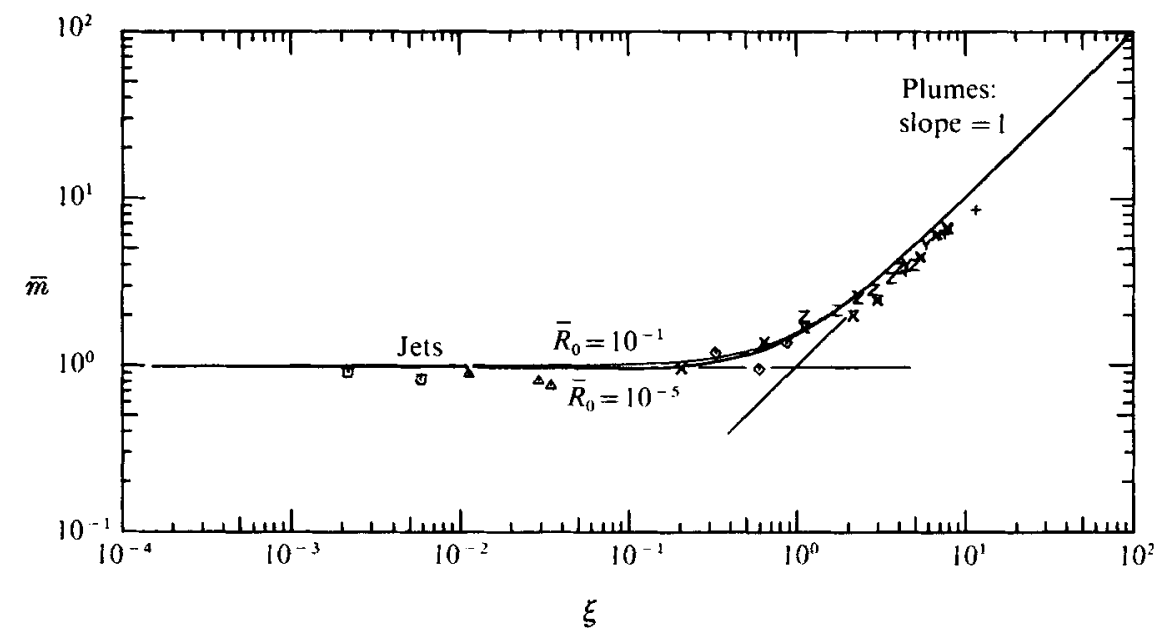

Fraure 4. Dimensionless local jet momentum flux as a function of scaled dimensionless distance from the jet origin (see caption to figure 3 for symbols). Curves are from (2.32).

The difference between the behaviour of the width parameter $C(x)$ and the jet Richardson number $R(x)$ is apparent from figure $3(b)$. It is very clear that the local Richardson number is asymptotic to an apparently constant value for values of $x-x_{0}$ large compared with the length scale $m_{0} / \beta_{0}^{\frac{2}{3}}$. A reasonable value for this constant, $R_{p}$, is about $0 \cdot 63$. For small values of the parameter $\left(x-x_{0}\right) \beta_{0}^{\frac{2}{3}} /$ $m_{0}$ the local Richardson number has a slope of $\frac{3}{2}$, as predicted by (2.37).

With the values of $C_{p}$ and $R_{p}$ now defined, the data given in table 2 can be plotted as functions of the scaled dimensionless variable $\xi$ and the results compared with the predictions given by (2.32), (2.33) and (2.35). Figure 4 is a graph of normalized momentum flux vs. $\xi$ and it can be seen that the agreement with (2.32) is reasonable although there is a slight momentum defect both in the jet and in the plume regime; Kotsovinos (1975) has discussed this in some detail. The data on the dimensionless volume flux, given in figure 5 , show remarkable 


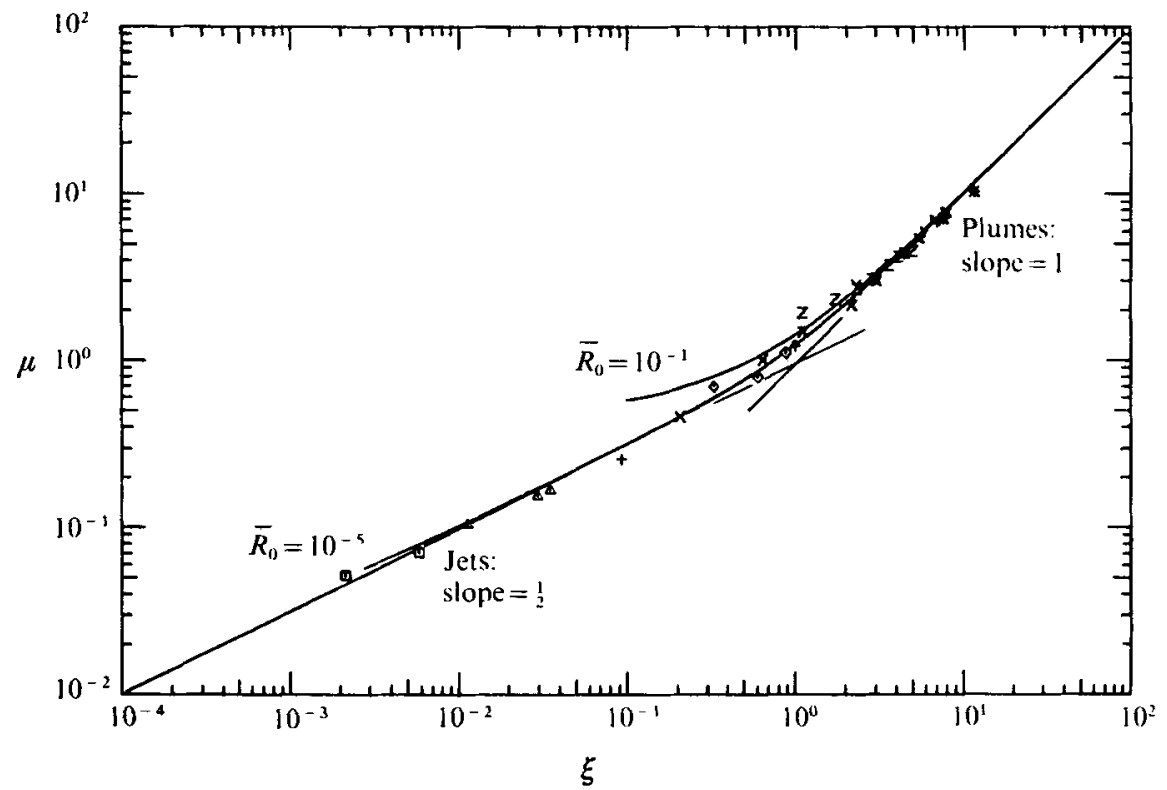

Figure 5. Dimensionless local jet volume flux (2.33) as a function of scaled dimensionless distance from the jet origin. Initial Richardson number $R_{0}:+, 0.00012 ; \Delta 0 \cdot 033 ; *, 0 \cdot 7$. (See caption to figure 3 for remainder of symbols.)

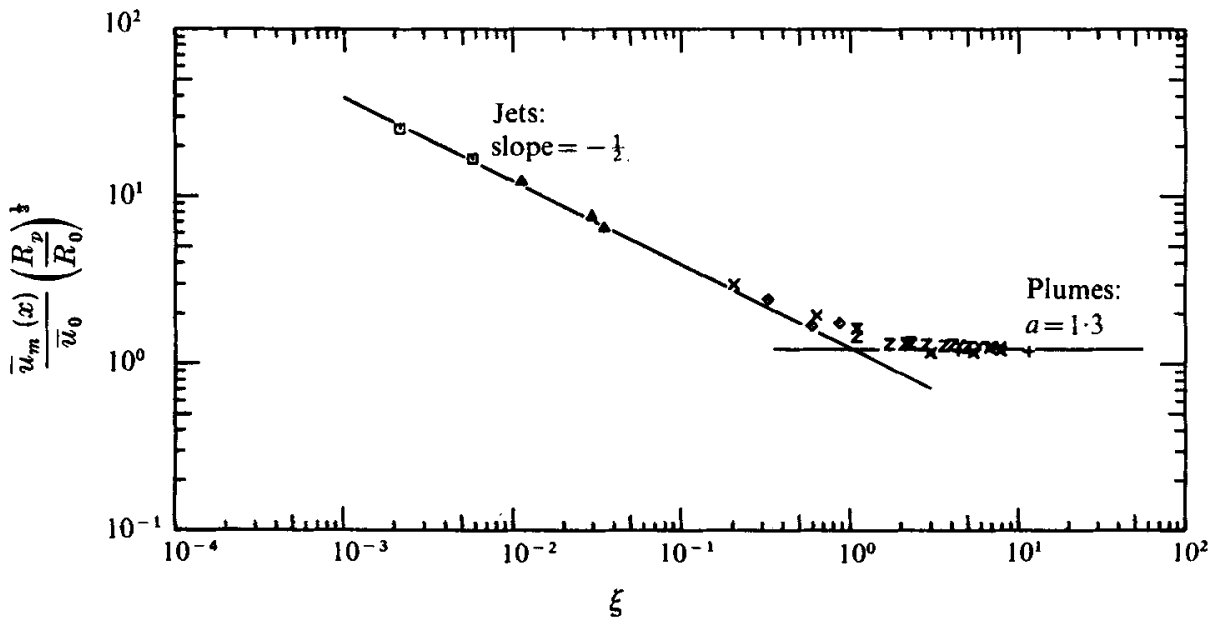

Figure 6. Dimensionless time-averaged velocity (2.35) on the jet axis as a function of scaled dimensionless distance from the jet origin (see caption to figure 3 for symbols).

agreement with the prediction of (2.33). The data on the axial mean velocity, shown in figure 6 , indicate that the empirical constant $a$ in $(2.35)$ has a value of about $1 \cdot 3$.

These results appear to indicate that the approximation $k=\frac{1}{2}$, i.e. assuming equal values of the dimensionless variables $C_{j}$ and $C_{p}$, is a worthwhile approach to the theory of plane buoyant jets. 


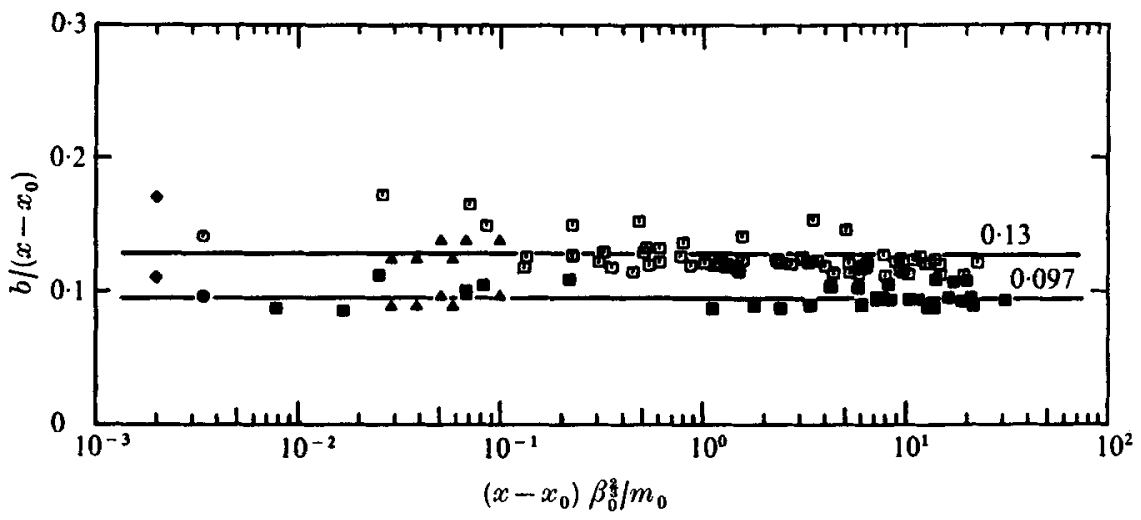

Figure 7. Jet angle based on mean velocity half-width (solid symbols) and mean temperature half-width (open symbols) as a function of dimensionless distance from the jet origin. $\square$, Kotsovinos (1975); $\bigcirc$, Jenkins \& Goldschmidt (1973); $\triangle$, van der Hegge Zijnen (1958); $\diamond$, Reichardt (1942). Solid lines are empirical.

The experimental results may be used to investigate one further mean feature of turbulent jet and plume flows. There has been some discussion in the literature concerning the ratio of the half-widths of the mean temperature and velocity profiles. Since only 13 simultaneous measurements of velocity and temperature profiles were carried out we have plotted in figure 7 the two ratios

$$
b_{u}(x) /\left(x-x_{0}\right), \quad b_{T^{\prime}}(x) /\left(x-x_{0}\right)
$$

against the scaled distance $\left(x-x_{0}\right) \beta_{0}^{\frac{2}{3}} / m_{0}$ for all the temperature and velocity profiles measured. The virtual origins used in each case are those obtained from the respective velocity or temperature half-widths given in table 1 or 2 . Although there is a fair degree of scatter in the data it does appear that both $b_{u}(x)$ and $b_{T}(x)$ are, to within a first approximation, linear functions of $x-x_{0}$ and that they have a common ratio for both jets and plumes. The graph also includes halfwidth data from experiments in turbulent jets with little buoyancy by Jenkins \& Goldschmidt (1973), van der Hegge Zijnen (1958) and Reichardt (1942). It can be seen that these previous experiments are in reasonable agreement with the results of this study. An acceptable value for $\lambda=b_{T} / b_{u}$ appears to be about $1 \cdot 35$ (to be compared with a value of 1.16 for round jets and plumes) although a declining trend does seem to occur in the temperature data.

\section{Heat flux}

Since the heat flux in a buoyant jet in a homogeneous environment must be conserved it becomes possible to determine the flux of heat transported by the turbulent fluctuations in the jet. We know that the initial heat flux is

$$
\begin{aligned}
H_{0} & \left.=\rho c_{p} \int_{-B(x)}^{+B(x)} \overline{u(x, y, t) T(x, y, t}\right) d y \\
& =\rho c_{p} \int_{-B(x)}^{+B(x)}\left(\bar{u}(x, y) \bar{T}(x, y)+\overline{u^{\prime} T^{\prime}}\right) d y \\
& =H_{l}+H_{T},
\end{aligned}
$$




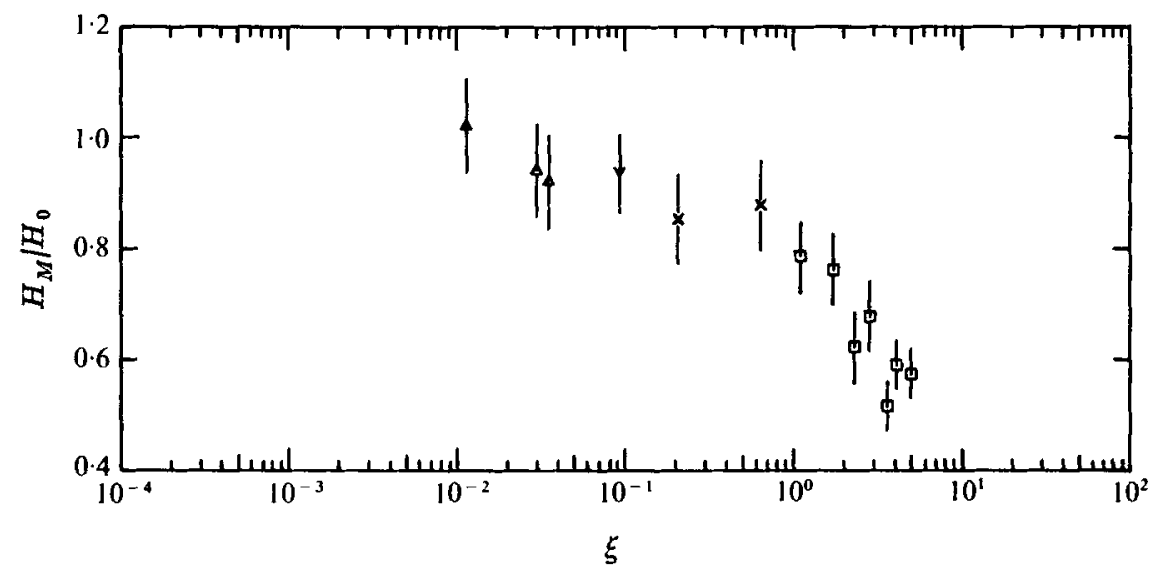

Figure 8. Relative fraction of total heat transfer due to mean advection as a function of the scaled dimensionless distance from the jet origin. Initial Richardson number $R_{0}$ : $\triangle, 0.00006 ; \mathrm{Y}, 0.00012 ; \mathrm{X}, 0.002 ; \square, 0.17$.

where $c_{p}$ is the specific heat of the liquid at constant pressure, $H_{M}$ the heat flux transported by the mean motion and $H_{T}$ the turbulent heat flux. The profiles of both mean velocity and temperature were measured in 13 different cross-sections, so that using the exponential curves fitted to the experimental data it is possible to calculate $H_{M I}$ and therefore deduce $H_{T}$. Figure 8 is a plot of the ratio $H_{M} / H_{0}$ as a function of the non-dimensional distance $\xi$. It should be noted, however, that there is a substantial margin for error in the calculation since the computation of the ratio $H_{M} / H_{0}$ involves additive relative errors in the measurement of $\bar{u}_{m}(x)$, $\bar{T}_{M}(x), b_{T}(x), b_{u}(x), \bar{u}_{0}$ and $\bar{T}_{0}$. Estimates of the possible experimental error are shown by error bars. Even with the possible error margins shown it is apparent that there is a distinct change in the fraction of the heat flux carried by the turbulence as the flow becomes more plume-like. This distinct change in the manner in which the heat flux is transported is not surprising: it seems likely that in a plume, which is a buoyancy-driven flow, the velocity and temperature fluctuations will be highly correlated, leading to a significant increase in the turbulent transport. This result calls into question one of the basic hypotheses that has been made in the integral theory of buoyant jets, namely that the turbulent flux of buoyancy can be ignored in a turbulent buoyant jet.

Since the results presented are probably the first such measurements of the mean flux in a plume additional, confirmatory studies are probably warranted.

The authors gratefully acknowledge the support of this work by the U.S. National Science Foundation, more recently through Grant no. ENG75-02985 and earlier by Grant no. GK-35774X. 


\section{REFERENCES}

Bradbury, L. 1965 The structure of the self-preserving turbulent plane jet. J. Fluid Mech. 23, 31-64.

Brooks, N. H. \& Kor, R. C. Y. 1965 Discharge of sewage effluent from a line source into a stratified ocean. 11th Cong. Int. Assoc. Hydraul. Res. paper 2.19 .

Cormack, D. E. 1975 Studies of a phenomenological turbulence model. Ph.D. thesis, California Institute of Technology, Pasadena.

Corrsin, S. \& UbEroI, M. 1950 Further experiments on the flow and heat transfer in a heated turbulent air jet. N.A.C.A. Rep. no. 998.

Crow, S. C. \& Champagne, F. H. 1971 Orderly structure in jet turbulence. J. Fluid Mech. 48, 547-591.

HEGGE ZIJNEN, B. G. VAN DER 1958 Measurements of the distribution of heat and matter in a plane turbulent jet of air. Appl. Sci. Res. 7, 277-292.

Jenkins, P. E. \& Goldschmidt, V. W. 1973 Mean temperature and velocity in a plane turbulent jet. Trans. A.S.M.E., J. Fluids Engng 95, 581-584.

Kotsovinos, N. E. 1975 A study of the entrainment and turbulence in a plane buoyant jet. Ph.D. thesis, California Institute of Technology, Pasadena.

LeE, S. L. \& Emmons, H.W. 1961 A study of natural convection above a line fire. J. Fluid Mech. 11, 353-368.

List, E. J. \& Imberger, J. 1973 Turbulent entrainment in buoyant jets and plumes. J. Hydraul. Div., Proc. A.S.C.E. 99 (HY9), 1461-1474.

List, E. J. \& Imberger, J. 1975 Closure of discussion to 'Turbulent entrainment in buoyant jets and plumes'. J. Hydraul. Div., Proc. A.S.C.E. 101 (HY5), 617-620.

LUMLEY, J. L. \& KHAJEH-NoURI, B. 1974 Computational modeling of turbulent transport. Adv. in Geophysics, 18A: 162-192.

Milder, D. R. \& Comings, E. W. 1957 Static pressure distribution in the free turbulent jet. J. Fluid Mech. 3, 1-16.

Morron, B. R. 1959 Forced plumes. J. Fluid Mech. 5, 151-163.

Morton, B., TaYlor, G. I. \& Turner, J. S. 1956 Turbulent gravitational convection from maintained and instantaneous sources. Proc. Roy. Soc. A 234, 1-23.

Priesteey, C. H. B. \& BALL, F. K. 1955 Continuous convection from an isolated source of heat. Quart. J. Roy. Met. Soc. 81, 144-157.

Reichardt, H. 1942 Gesetzmassigkeiten der freien Turbulenz. V'DI Forschungsheft 13, $1-22$.

Rouse, H., YIH, C.-S. \& Humphreys, H. W. 1952 Gravitational convection from a boundary source. Tellus, 4, 201-210.

Saffman, P. G. 1970 A model of inhomogeneous turbulent flow. Proc. Roy. Soc. A 317, 417-433.

Schlichting, H. 1960 Boundary Layer Theory, 4th edn. McGraw-Hill.

Stevenson, W. 1970 Optical frequency shifting by means of a rotating diffraction grating. Appl. Optics 9, 649-652.

StewaRT, R. W. 1956 Irrotational motion associated with free turbulent flows. J. Fluid Mech. 1, 593-606.

TAYLOR, G. I. 1932 The transport of vorticity and heat through fluids in turbulent motion. Proc. Roy. Soc, A 135, 685-702.

TAYLOR, G. I. 1958 Flow induced by jets. J. Aero. Sci. 25, 464-465.

TOLLMiEn, W. 1926 Die Berechnung turbelenter Ausbreitungsvorgange. Z. angew. Math. Mech. 6, 468-478.

Townsend, A. A. 1956 The Structure of Turbulent Shear Flow. Cambridge University Press. 